\title{
Colgajo perforante de la arteria circunfleja ilíaca superficial para la cobertura de defectos en los miembros
}

\author{
Sebastián E. Valbuena, ${ }^{*}$ Emanuel Pereira, ${ }^{*}$ Braian Conde** \\ "Servicio de Ortopedia y Traumatología, Hospital El Cruce Alta Complejidad en Red “Dr. Néstor C. Kirchner”, Florencio Varela, \\ Buenos Aires, Argentina \\ **Servicio de Ortopedia y Traumatología, Hospital “Dr. Alejandro Korn”, Melchor Romero, Buenos Aires, Argentina
}

\begin{abstract}
RESUMEN
El colgajo perforante de la arteria circunfleja ilíaca superficial se utiliza para la reconstrucción de los miembros, sobre todo, en el dorso de las manos y los pies, en la zona de flexión articular y en cirugía maxilofacial, porque es un colgajo fino, flexible y versátil. Presentamos tres pacientes: dos casos pediátricos para la reconstrucción de secuelas traumáticas de la mano y el dorso del pie, y un adulto para la reconstrucción de una quemadura del dorso de la mano. Conclusiones: El colgajo perforante de la arteria circunfleja ilíaca superficial es ideal para el dorso de la mano, el pie y los pliegues articulares. Pese a las variaciones anatómicas en el nacimiento de la arteria circunfleja ilíaca superficial, el dominio de las técnicas microquirúrgicas y de la supermicrocirugía permite tomar, con seguridad, un colgajo de dimensiones moderadas o grandes, fino, con baja morbilidad en la zona dadora.

Palabras clave: Colgajo perforante; arteria circunfleja ilíaca superficial; perforosoma; inguinal.
\end{abstract}

Nivel de Evidencia: $\mathrm{V}$

\section{Superficial Circumflex Iliac Artery Perforator Flap for the Coverage of Limb Defects}

\section{ABSTRACT}

The superficial circumflex iliac artery perforator (SCIP) flap is a thin, pliable, and versatile flap used mainly for extremities and head and neck reconstruction. We presented 3 cases of SCIP flap reconstructions. Two pediatric cases for the reconstruction of traumatic sequelae of the hand and dorsal foot. One adult case for the reconstruction of a severe burn of the dorsal hand. Conclusion: The SCIP flap is ideal for the reconstruction of the dorsal aspect of the hand, foot, and flexion crease. Despite the anatomical variations in the origin of the superficial circumflex iliac artery, the use of microsurgical techniques and super-microsurgery allows safe harvesting of a thin flap of moderate to large dimensions, with low morbidity in the donor area.

Key words: Perforator flap; SCIP; circumflex iliac superficial artery; perforosome; inguinal.

Level of Evidence: $\mathrm{V}$

\section{INTRODUCCIÓN}

El colgajo inguinal basado en la arteria circunfleja ilíaca superficial (ACIS) fue descrito por McGregor y Jackson, en 1972, para la cobertura de defectos en el miembro superior. Este colgajo es fácil de realizar, una de sus desventajas es que el miembro debe estar unido a la región pélvica como mínimo tres semanas, y requiere de otro tiempo quirúrgico para su separación. ${ }^{1}$ En 1973, Daniel y Taylor propusieron su uso de forma libre ${ }^{2}$ y, aunque una de sus características atractivas es la baja morbilidad en la zona dadora, este colgajo pierde popularidad frente a otros colgajos libres debido a sus variaciones anatómicas.

A partir del auge de los colgajos perforantes que permiten diseñar colgajos de gran dimensión desde una arteria perforante, es que Koshima y cols. describen el colgajo perforante libre de la ACIS para la reconstrucción de miembros, ${ }^{3}$ y se convierte en un colgajo muy útil para las reconstrucciones maxilofaciales y del área pudenda.

Recibido el 2-12-2019. Aceptado luego de la evaluación el 4-8-2020 • Dr. SEBASTIÁN E. VALBUENA • valbuena.sebastian@gmail.com https://orcid.org/0000-0002-0209-1554 Cómo citar este artículo: Valbuena SE, Pereira E, Conde B. Colgajo perforante de la arteria circunfleja ilíaca superficial para la cobertura de defectos en los miembros. Rev Asoc Argent Ortop Traumatol 2021;86(6):812-820. https://doi.org/10.15417/issn.1852-7434.2021.86.6.1035 
La posibilidad de tomar colgajos finos, sin necesidad de un desgrasado posterior posiciona al colgajo perforante de la ACIS dentro de las elecciones para la cobertura de miembros, sobre todo, en el dorso de la mano, del pie y los pliegues articulares.

El objetivo de este artículo es describir las ventajas del colgajo de la ACIS y comunicar la resolución de tres casos clínicos.

\section{CASO CLÍNICO 1}

Varón de 6 años, que sufrió un accidente en la panadería de sus padres con la máquina sobadora de masa, que le provocó un desguantamiento completo del pulgar y el dorso de la mano, una amputación tipo III de la clasificación de Allen y Dautel (distal a la articulación interfalángica). Fue tratado mediante diferentes cirugías en un hospital local. Un año después del accidente, acudió a nuestra consulta. En la evaluación, se observó el pulgar totalmente incluido dentro de la mano y ausencia de la mitad de la falange distal del pulgar (Figura 1).

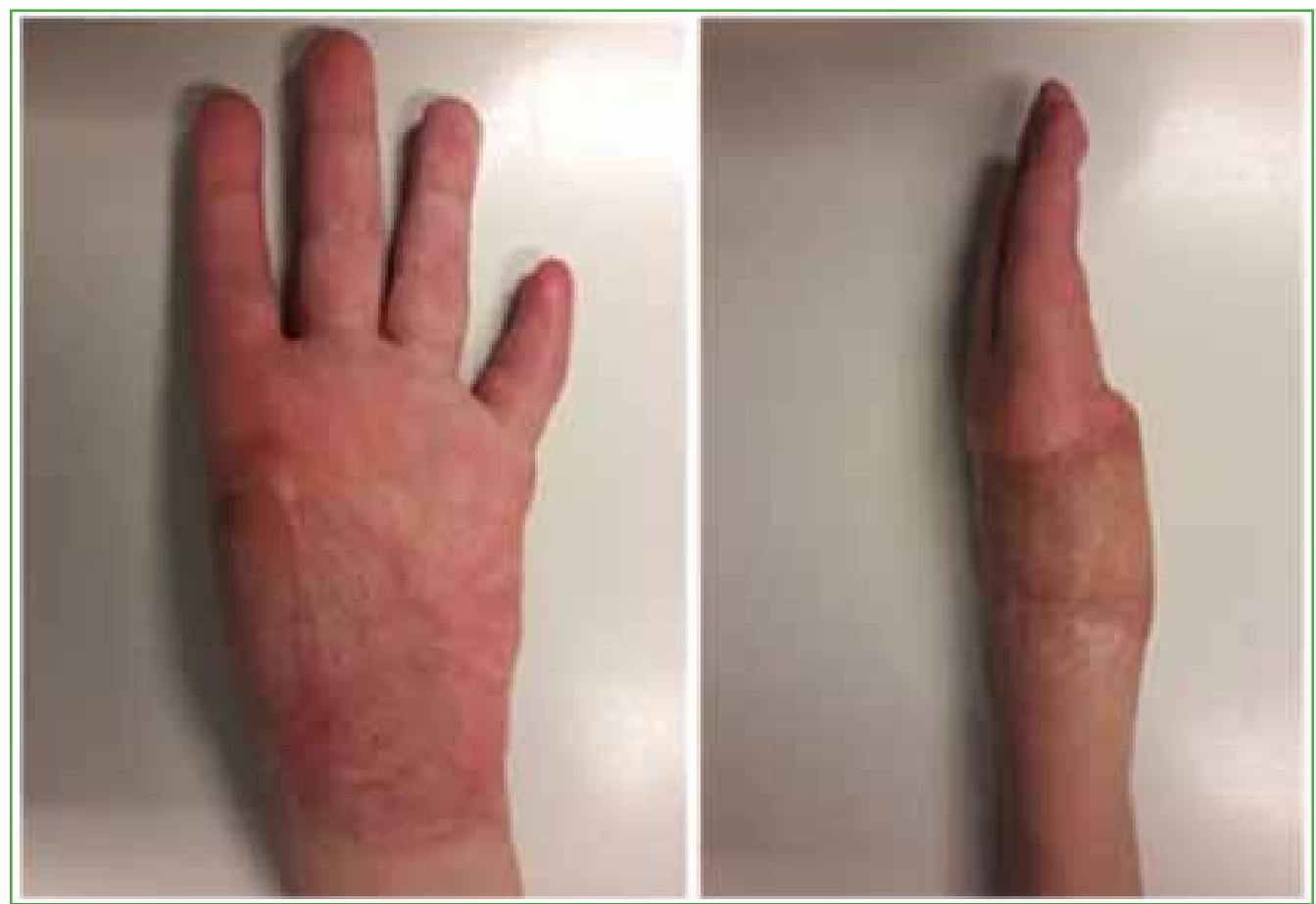

Figura 1. Secuela de herida por sobadora. Avulsión del pulgar con amputación distal a nivel de F2, tipo III, con inclusión completa del pulgar en la palma de la mano.

Se planificó una cirugía reconstructiva en dos tiempos. El primer tiempo consistió en la reconstrucción de la primera comisura con colocación del pulgar en una posición funcional. Se realizó la separación del pulgar con la apertura de la comisura, y el defecto cutáneo se reconstruyó con un colgajo inguinal de 6 x $12 \mathrm{~cm}$ basado en la rama profunda de la ACIS. Luego se procedió a la anastomosis término-lateral de la arteria cubital a nivel del canal de Guyon y a la anastomosis de la vena satélite término-terminal con una de las venas de la arteria cubital (Figura 2). 


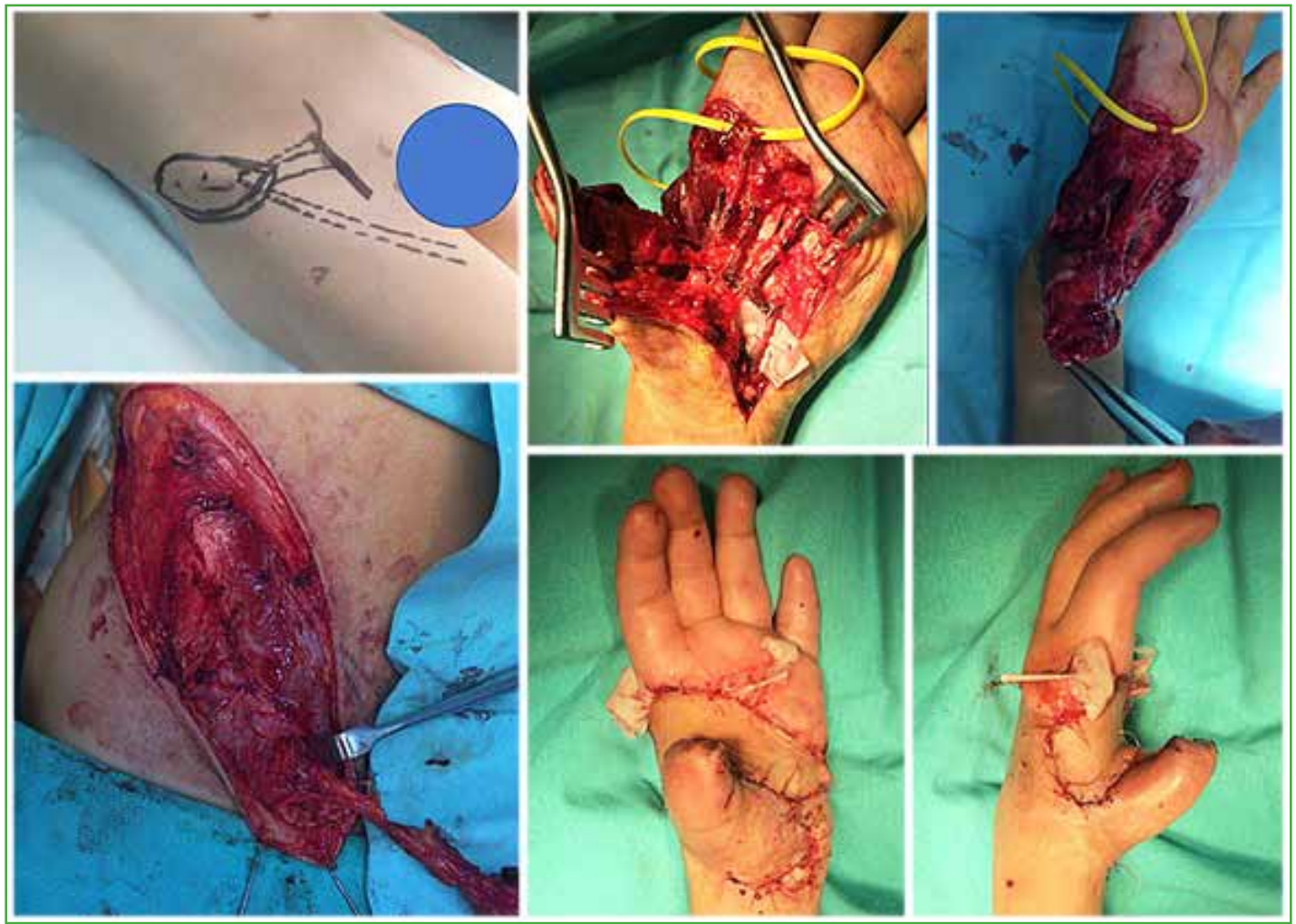

Figura 2. Separación del pulgar, apertura de la primera comisura, tallado de un colgajo de arteria circunfleja ilíaca superficial libre basado en la rama profunda de la arteria circunfleja ilíaca superficial.

Tras la rehabilitación de la movilidad del pulgar, a los ocho meses, se llevó a cabo el segundo tiempo quirúrgico que consistió en la reconstrucción del pulgar con una transferencia del hemihallux y anastomosis término-lateral a la arteria radial y dos venas satélite a venas del dorso de la mano (Figura 3).

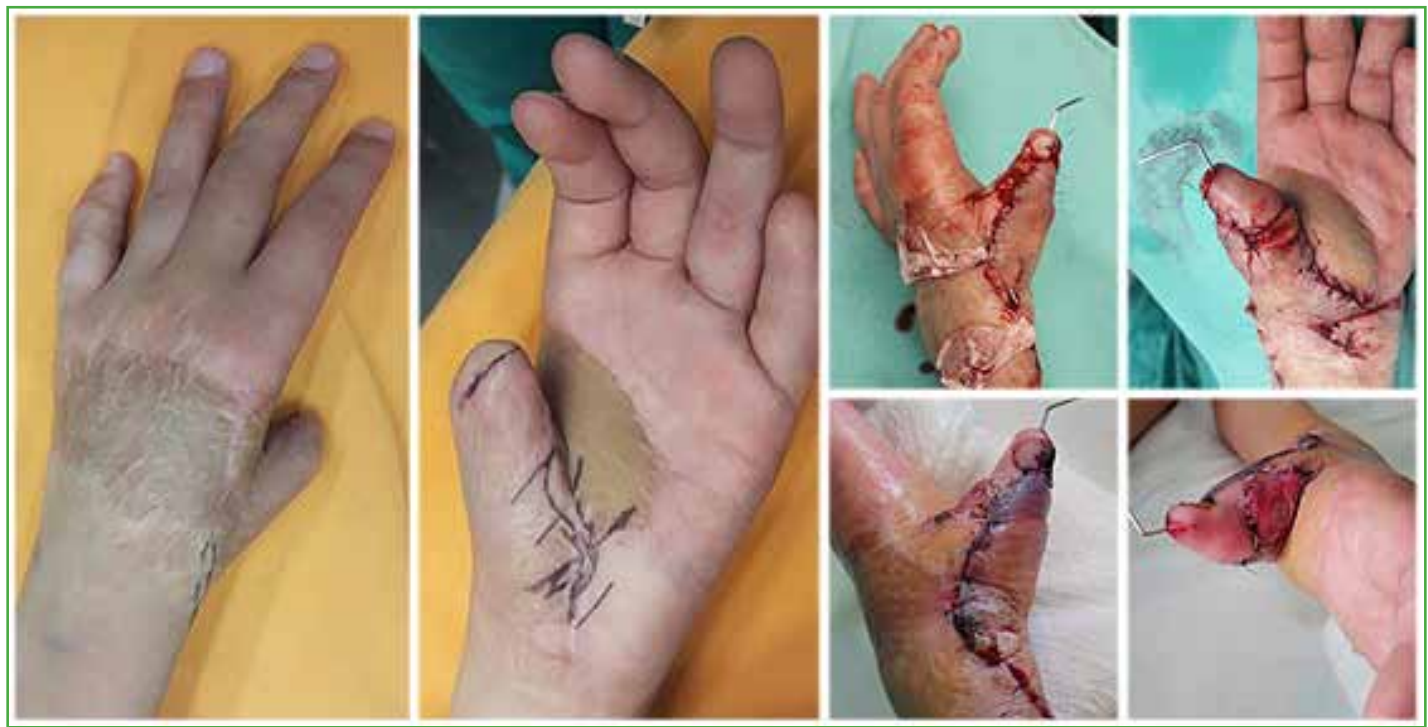

Figura 3. Segundo tiempo quirúrgico: reconstrucción del pulgar con transferencia libre del hemihallux. 
$\mathrm{Al}$ año de la cirugía, el aspecto estético y la función del pulgar (Kapandji 8) son buenos. La única complicación es una cicatriz queloide en el dorso de la muñeca (Figura 4).

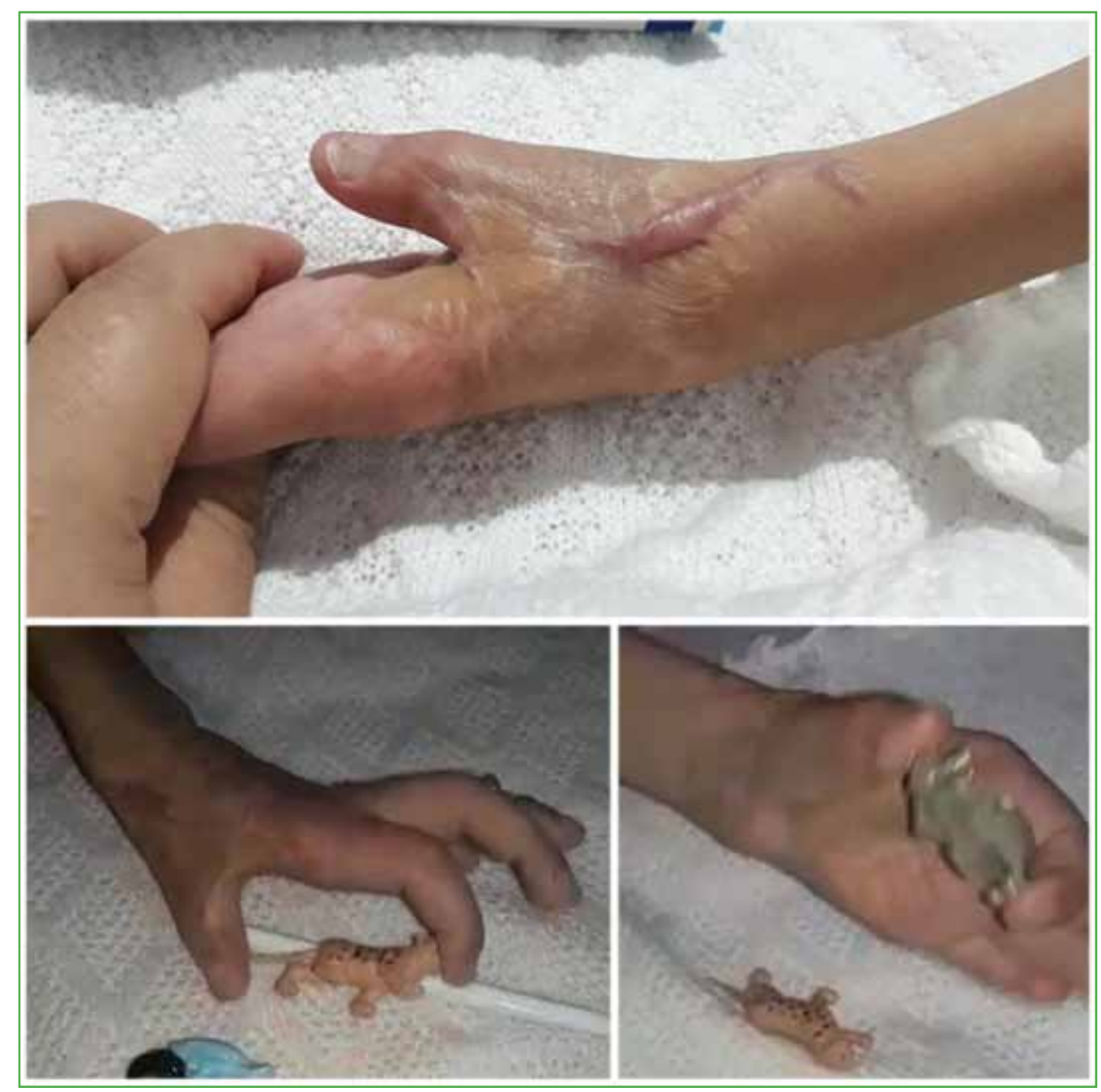

Figura 4. Excelente resultado estético y funcional del pulgar. Nótese la cicatriz queloide en la región dorsorradial de la muñeca.

\section{CASO CLÍNICO 2}

Mujer de 37 años, epiléptica, que sufre una quemadura profunda del dorso de la mano y los dedos durante una crisis. En la institución local, se le practicaron incisiones de descarga longitudinales para evitar el síndrome compartimental (Figura 5). 


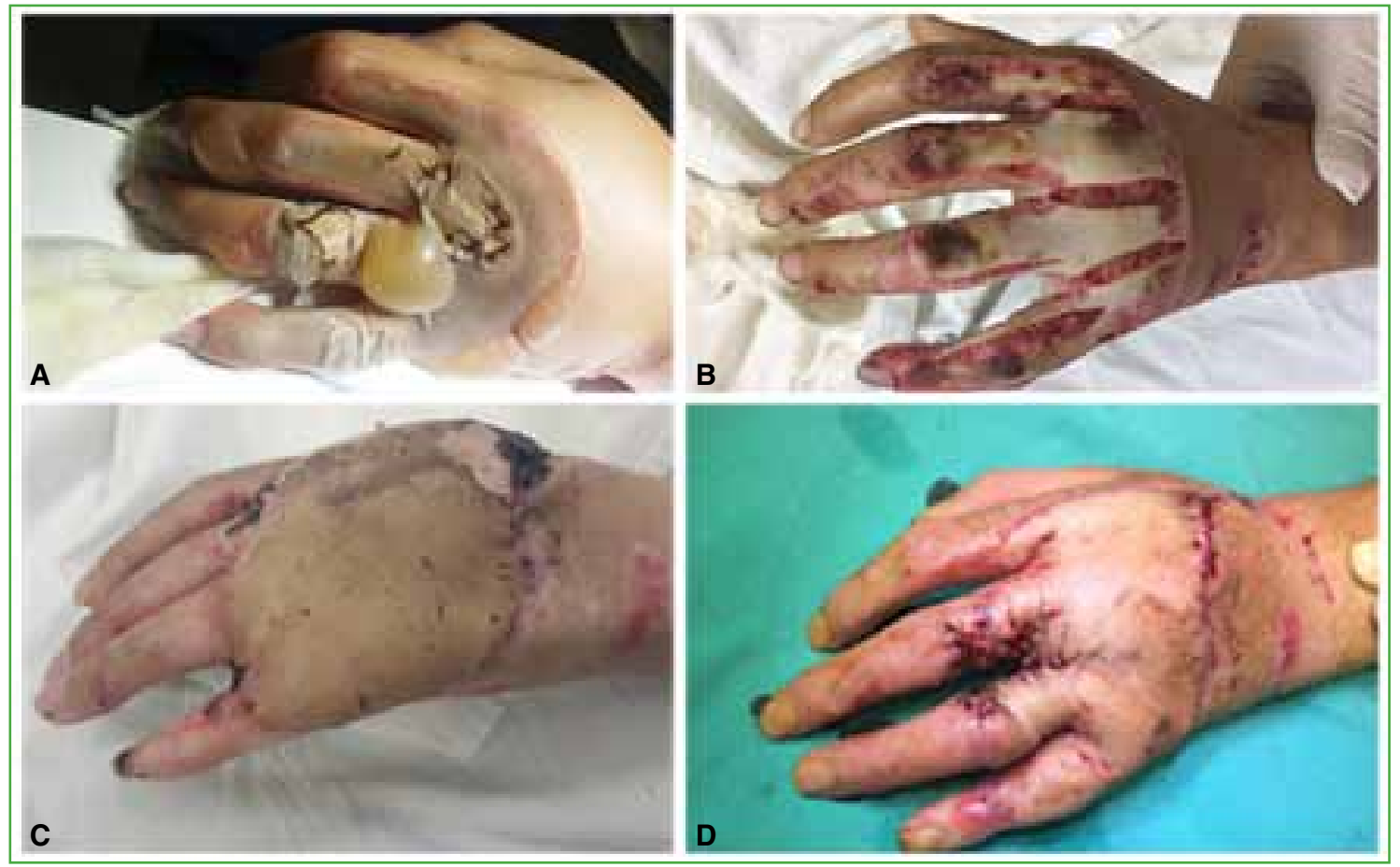

Figura 5. A. Quemadura profunda del dorso de la mano y los dedos. B. A los siete días de las incisiones longitudinales en el dorso de la mano y los dedos. C. Tres semanas después de la cirugía. D. A las cinco semanas del colgajo de la arteria circunfleja ilíaca superficial, se realiza la apertura de las comisuras.

Se planificó la resección del tejido patológico y la colocación de un colgajo de la ACIS fino, basado en la rama superficial, a las tres semanas de la quemadura. Debido a la necesidad de un pedículo largo para construir la anastomosis con la arteria radial, se talló un colgajo cutáneo de 14 × $7 \mathrm{~cm}$ distal, en el que se incluyó el pedículo de la perforante superficial en un colgajo graso. La anastomosis fue término-lateral a la arteria radial y dos venas a venas dorsales de la mano y la muñeca. El colgajo cubrió todo el defecto dorsal y de la región metacarpofalángica de los dedos. A las tres semanas, se realizó la separación de la sindactilia de la segunda y cuarta comisura y, a la quinta semana, la separación de la tercera comisura. Al octavo mes de la operación, la movilidad y la función de la mano eran completas, con un aspecto estético aceptable (Figura 6).


Figura 6. A los ocho meses de la cirugía, la movilidad de la mano es completa y el resultado estético es excelente. Colgajo fino que no necesita desgrasado posterior. 


\section{CASO CLÍNICO 3}

Niña de 5 años, que tiene una secuela de quemadura con agua hirviendo en el dorso del pie derecho con retracción cutánea y deformidad del hallux en extensión fija a $90^{\circ}$, de tres años de evolución. Fue sometida a múltiples Z-plastias y correcciones sin buen resultado. Desde hace 30 meses, la paciente no puede colocarse un calzado debido a la deformidad residual (Figura 7).

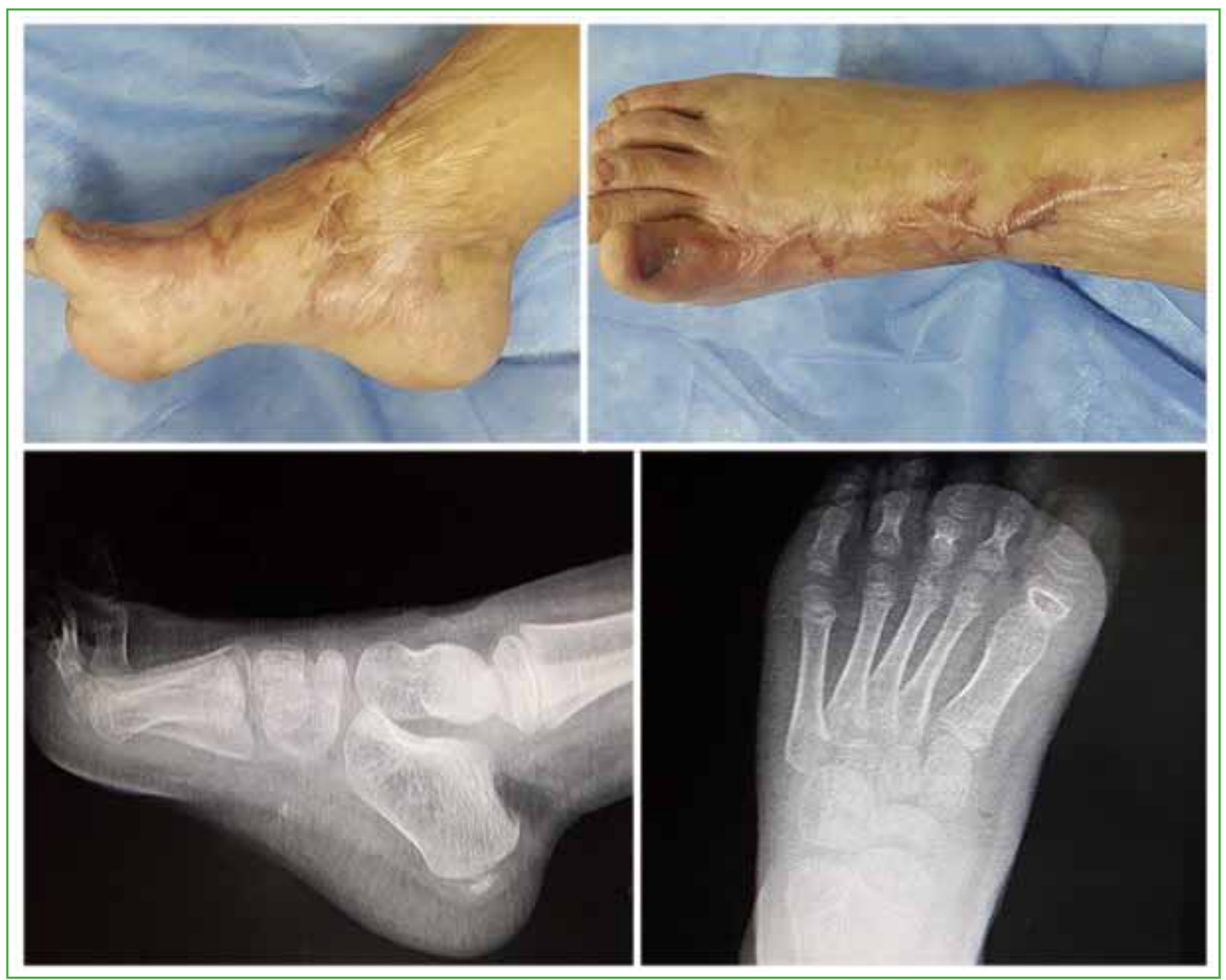

Figura 7. Secuela de quemadura. Retracción cutánea del dorso del pie con deformidad en extensión de la articulación metatarsofalángica fija en $90^{\circ}$.

Se procedió a la resección de la cicatriz, al alargamiento del extensor del hallux, la liberación capsular más la fijación transitoria del hallux en posición plantígrada. Se talló un colgajo de la ACIS de 6 x $13 \mathrm{~cm}$ basado en la rama superficial. Se realizó una anastomosis término-terminal a la arteria pedia y de dos venas una satélite y otra superficial. Se consiguió la corrección del hallux y, a los seis meses de la cirugía, la paciente deambula con calzado sin inconvenientes (Figura 8). 


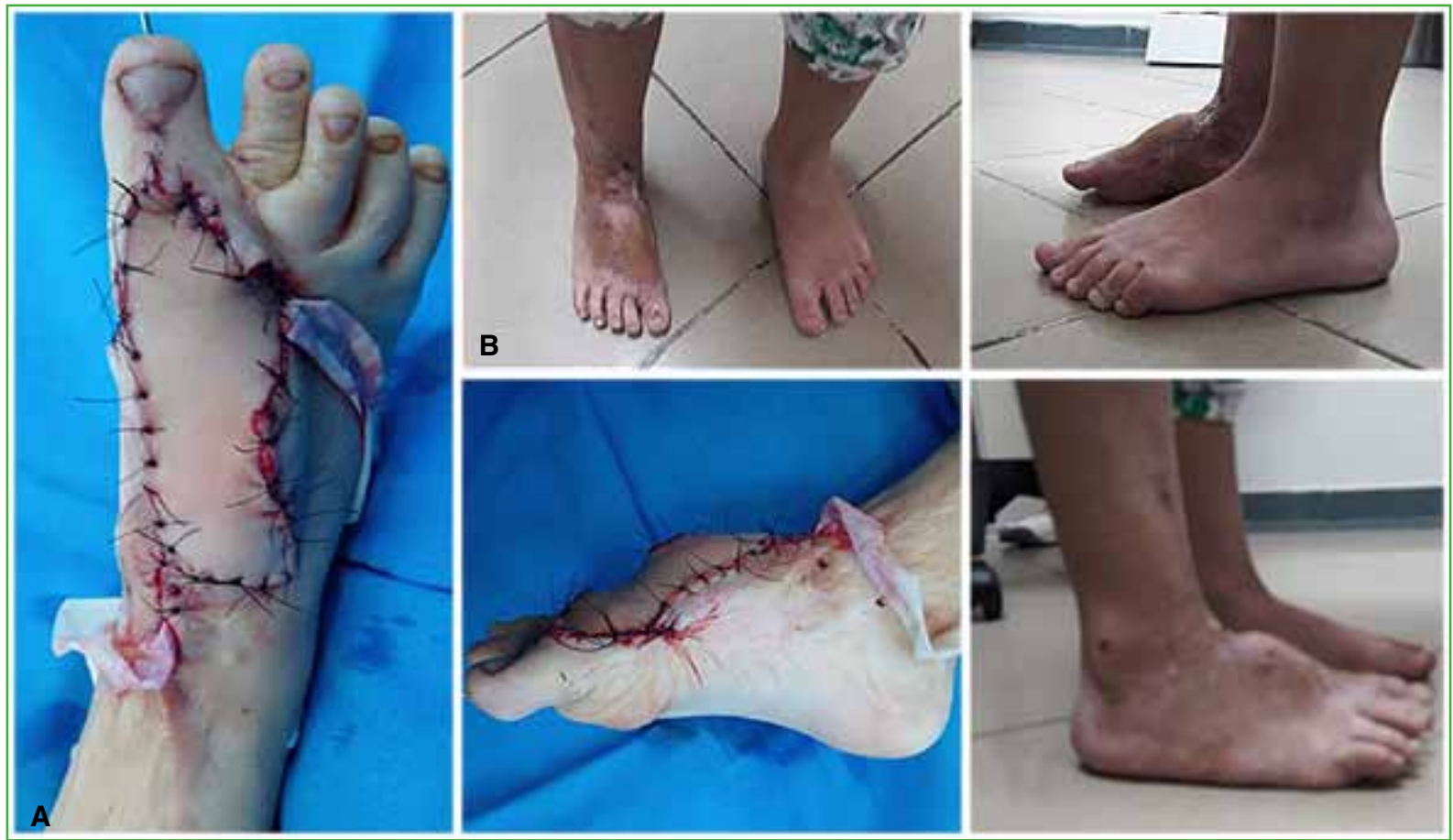

Figura 8. A. Resultado posoperatorio inmediato. B. Resultado a los cuatro meses, apoyo plantígrado del pie, sin molestias al usar calzado cerrado.

\section{DISCUSIÓN}

Los colgajos perforantes ya sea en hélice, locales o libres tienen múltiples ventajas debido a la baja morbilidad en el área donante y a su versatilidad para adaptarse al área receptora. ${ }^{4}$

Saint-Cyr y cols. demuestran que el área irrigada por una arteria perforante, denominada perforosoma, está conectada directa e indirectamente con otros perforosomas, lo que aumenta el área de un colgajo a partir de estas anastomosis. La piel es irrigada por anastomosis directas y anastomosis indirectas del plexo subdérmico. Por lo tanto, un colgajo de grandes dimensiones puede ser tomado irrigado por una sola perforante mientras se mantenga la red indirecta subdérmica. ${ }^{5}$

A su vez, debido a estas anastomosis subdérmicas es factible tomar colgajos finos realizando disecciones superficiales. ${ }^{6,7}$ Son ideales para pliegues articulares, lesiones en las manos y el dorso del pie, y mejoran considerablemente la estética y evitan el desgrasado posterior. ${ }^{8}$ Con el colgajo de la ACIS superfino de menos de $5 \mathrm{~mm}$ de espesor para defectos de talla moderada, se ha logrado una tasa de éxito superior al $90 \%{ }^{7,9}$

La ACIS nace de la arteria femoral en el $83,5 \%$ de los casos, de la femoral superficial en el $8 \%$, de la femoral profunda en el $8 \%$ y de la circunfleja lateral profunda en el $1 \% .^{10}$ Asimismo, hay descripciones anatómicas que señalan que comparten un tronco común con la arteria epigástrica superficial en el 20-30,4\% de los pacientes. ${ }^{11,12}$ Un centímetro distal a su nacimiento se divide en dos ramas que corren superior y lateral dirigiéndose a la espina ilíaca anterosuperior. ${ }^{10}$ La rama superficial, perforante medial cutánea, penetra la fascia profunda y se dirige directamente hacia la piel. La rama profunda que discurre por debajo de la fascia del sartorio, al cual irriga, da una perforante cutánea medial, y una perforante lateral en el borde externo del sartorio, luego continua por debajo del nervio femoral cutáneo ${ }^{8,10}$ La rama profunda de la ACIS da ramas para el músculo sartorio, el nervio femoral cutáneo y la cresta ilíaca. ${ }^{13}$ Una ventaja de esta perforante profunda es que permite tomar un pedículo más largo y, debido a su red anastomótica profunda, obtener un colgajo quimérico con músculo y hueso (Figura 9). 


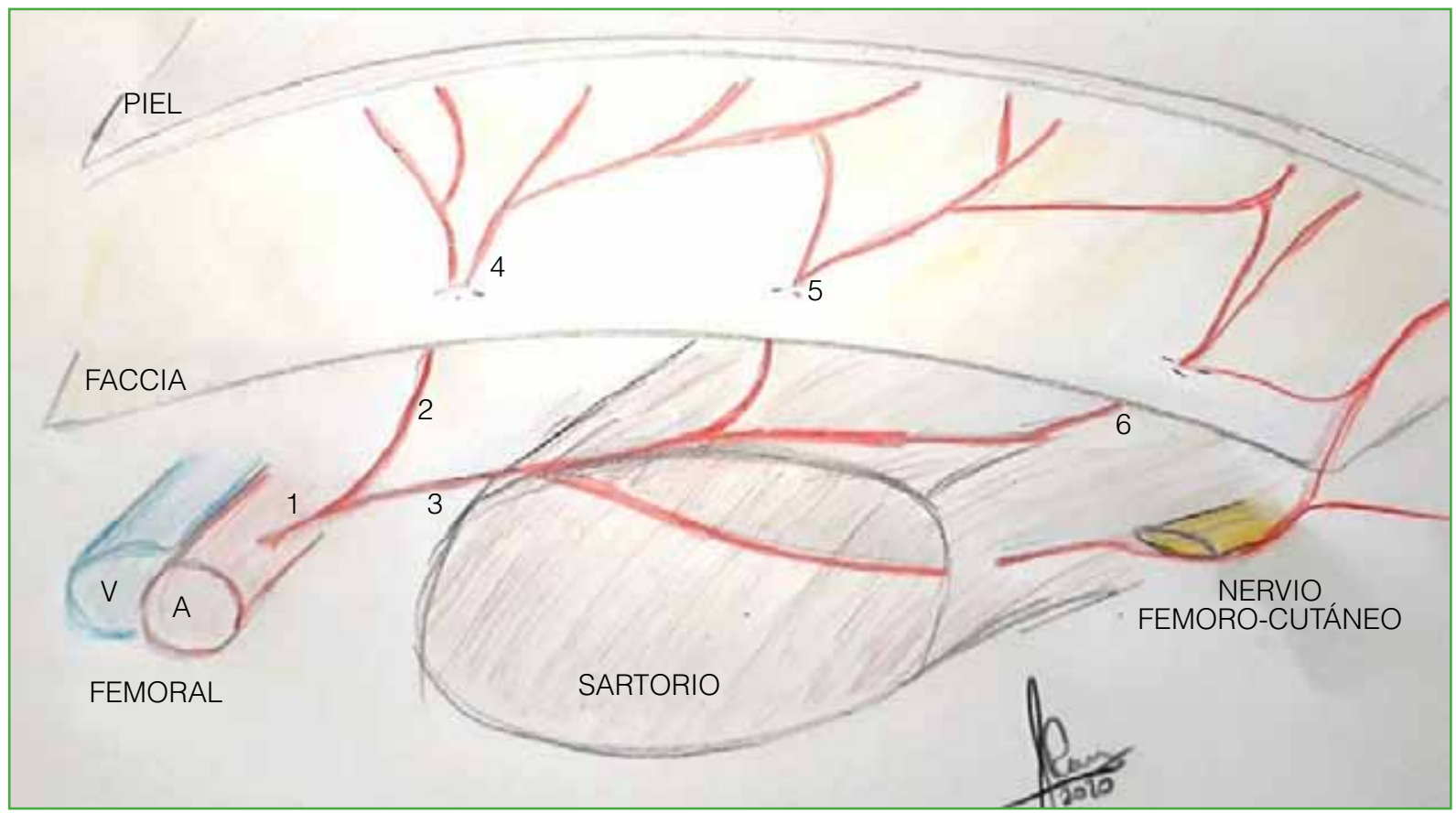

Figura 9. Esquema de la irrigación: 1 = arteria circunfleja ilíaca superficial, 2 = rama superficial de la arteria circunfleja ilíaca superficial, 3 = rama profunda de la arteria circunfleja ilíaca superficial, 4 = perforante medial cutánea de la rama superficial, $5=$ perforante medial cutánea de la rama profunda, $6=$ perforante lateral de la rama profunda.

La rama superficial presenta una disección más simple y su diámetro suele ser mayor. ${ }^{10}$ Esta arteria es una rama de la ACIS en el 96\%, pero su origen puede ser la arteria epigástrica, la arteria femoral superficial o la arteria pudenda superficial. Cuando esto ocurre y se ha diseñado un colgajo basado en la rama superficial, es necesario realizar una anastomosis de perforante a perforante utilizando técnicas de supermicrocirugía. Shu y cols. comunicaron que también hay una concordancia del $100 \%$ entre los hallazgos quirúrgicos y la angiotomografía en relación con el origen de esta arteria, y aconsejan realizar este estudio, de forma sistemática, para prever el origen arterial. A su vez, estos autores describen que la rama superficial de la ACIS penetra la fascia profunda en un área de 4,2 x 2 $\mathrm{cm}$, centrada a $4,5 \mathrm{~cm}$ lateral y $1,5 \mathrm{~cm}$ superior del tubérculo del pubis. Sostienen que la inclusión de esta área en el diseño del colgajo permite incluir a la rama superficial de la ACIS. ${ }^{10}$

Una perforante dominante de la rama superficial o profunda es suficiente para nutrir el colgajo. ${ }^{14}$ Sinna y cols. recomiendan tomar la rama profunda de la ACIS ${ }^{15}$ sin embargo, Shu y cols. enfatizan que la perforante superficial es suficiente para la irrigación del colgajo. ${ }^{10}$ del Piñal y cols. postulan que es suficiente la inclusión de la perforante superficial de la ACIS y la primera perforante cutánea medial de la rama profunda de la ACIS para colgajos de dimensiones grandes $(8 \times 25 \mathrm{~cm}){ }^{8}$

La toma de grandes colgajos irrigados solo por la rama superficial de la ACIS puede ocasionar la pérdida parcial del colgajo, la perforante superficial tiene dos formas de terminación en la piel: una con flujo axial en red y otra con una terminación en ancla, ${ }^{16}$ esta última variante es la que podría condicionar la pérdida parcial en los colgajos de grandes dimensiones.

Consideramos que la rama superficial es suficiente para diseñar colgajos de tamaño moderado, dejando la rama profunda para cuando se necesitan dimensiones mayores, un pedículo vascular largo o colgajos quiméricos.

\section{CONCLUSIONES}

El colgajo perforante de la ACIS es ideal para el dorso de la mano, el pie y los pliegues articulares. A pesar de las variaciones anatómicas en el nacimiento de la ACIS, el dominio de las técnicas microquirúrgicas y de la supermicrocirugía permite tomar, con seguridad, un colgajo de dimensiones moderadas a grandes, fino, con baja morbilidad en la zona dadora. 
Conflicto de intereses: Los autores no declaran conflictos de intereses.

ORCID de E. Pereira: https://orcid.org/0000-0001-8096-4114

ORCID de B. Conde: https://orcid.org/0000-0002-4504-5128

\section{BIBLIOGRAFÍA}

1. McGregor IA, Jackson IT. The groin flap. Br J Plast Surg 1972;25(1):3-16. https://doi.org/10.1016/s0007-1226(72)80003-1

2. Daniel RK, Taylor GI. Distant transfer of an island flap by microvascular anastomoses: A clinical technique. Plast Reconstr Surg 1973;52(2):111-7. https://doi.org/10.1097/00006534-197308000-00001

3. Koshima I, Nanba Y, Tsutsui T, Takahashi Y, Urushibara K, Inagawa K, et al. Superficial circumflex iliac artery perforator flap for reconstruction of limb defects. Plast Reconstr Surg 2004;113(1):233-40. https://doi.org/10.1097/01.PRS.0000095948.03605.20

4. Kim JT, Kim SW. Perforator flap versus conventional flap. J Korean Med Sci 2015;30(5):514-22. https://doi.org/10.3346/jkms.2015.30.5.514

5. Saint-Cyr M, Wong C, Schaverien M, Mojallal A, Rohrich RJ. The perforasome theory: vascular anatomy and clinical implications. Plast Reconstr Surg 2009;124(5):1529-44. https://doi.org/10.1097/PRS.0b013e3181b98a6c

6. Hong JP, Choi DH, Suh H, Mukarramah DA, Tashti T, Lee K, et al. A new plane of elevation: the superficial fascial plane for perforator flap elevation. J Reconstr Microsurg 2014;30(7):491-6. https://doi.org/10.1055/s-0034-1369807

7. Jeong HH, Hong JP, Suh HS. Thin elevation: A technique for achieving thin perforator flaps. Arch Plast Surg 2018;45(4):304-13. https://doi.org/10.5999/aps.2017.01529

8. del Piñal F, García-Bernal FJ, Studer A, Ayala H, Cagigal L, Regalado J. Super-thinned iliac flap for major defects on the elbow and wrist flexion creases. J Hand Surg Am 2008;33(10):1899-1904. https;//doi.org/10.1016/j.jhsa.2008.09.023

9. Kim JH, Kim KN, Yoon CS. Reconstruction of moderate-sized distal limb defects using a superthin superficial circumflex iliac artery perforator flap. J Reconstr Microsurg 2015;31(9):631-5. https://doi.org/10.1055/s-0035-1558959

10. Suh HS, Jeong HH, Choi DH, Hong JP. Study of the medial superficial perforator of the superficial circumflex iliac artery perforator flap using computed tomographic angiography and surgical anatomy in 142 patients. Plast Reconstr Surg 2017;139(3):738-8. https://doi.org/10.1097/PRS.0000000000003147

11. Nam YS, Han SH, Paik DJ. Morphometrics of arterial supply for superficial circumflex iliac artery flap in Koreans. Korean Journal of Physical Anthropology 2006;19(1):111. https://doi.org/10.11637/kjpa.2006.19.1.1

12. Del Sol M, Vasquez B. Arteria circunfleja iliaca superficial. Importancia anátomo quirúrgica. Int J Morphol 2013;31(2):629-32. https://doi.org/10.4067/S0717-95022013000200046

13. Yoshimatsu H, Steinbacher J, Meng S, Hamscha UM, Weninger WJ, Tinhofer IE, et al. Superficial circumflex iliac artery perforator flap: An anatomical study of the correlation of the superficial and the deep branches of the artery and evaluation of perfusion from the deep branch to the sartorius muscle and the iliac bone. Plast Reconstr Surg 2019;143(2):589-602. https://doi.org/10.1097/PRS.0000000000005282

14. Koshima I, Nanba Y, Nagai A, Nakatsuka M, Sato T, Kuroda S. Penile reconstruction with bilateral superficial circumflex iliac artery perforator (SCIP) flaps. J Reconstr Microsurg 2006;22(3):137-42. https://doi.org/10.1055/s-2006-939957

15. Sinna R1, Hajji H, Qassemyar Q, Perignon D, Benhaim T, Havet E. Anatomical background of the perforator flap based on the deep branch of the superficial circumflex iliac artery (SCIP Flap): a cadaveric study. Eplasty 2010;10:11. PMID: 20090859

16. Goh TL, Park SW, Cho JY, Choi JW, Hong JP. The search for the ideal thin skin flap: superficial circumflex iliac artery perforator flap--a review of 210 cases. Plast Reconstr Surg 2015;135(2):592-601.

https://doi.org/10.1097/PRS.0000000000000951 